\section{(2)} OPEN ACCESS

\section{- Additional material is published online only. To view please visit the journal online (http://dx.doi.org/10.1136/ jnnp-2019-321321) \\ ${ }^{1}$ Neurology, Odense Universitetshospital, Odense, Denmark \\ ${ }^{2}$ Department of Clinical Research, University of Southern Denmark, Odense, Denmark ${ }^{3}$ Neurology, Hospital of South West Jutland, Esbjerg, Denmark ${ }^{4}$ The Department of Regional Health Research, University of Southern Denmark, Odense, Denmark \\ ${ }^{5}$ MS Alliance of Southern Denmark, Esbjerg, Denmark ${ }^{6}$ Value Based Medicine, Biogen Idec Inc, Cambridge, Massachusetts, USA}

\section{Correspondence to} Dr Tobias Sejbaek, Neurology, Hospital of South West Jutland, Esbjerg 5000, Denmark; Tobias. Sejbaek.Mathiesen@rsyd.dk

Received 30 May 2019 Revised 24 September 2019 Accepted 28 September 2019 Published Online First 13 October 2019

\section{SLinked}

- http://dx.doi.org/10.1136/ jnnp-2019-321615

\section{Check for updates}

(C) Author(s) (or their employer(s)) 2019. Re-use permitted under CC BY-NC. No commercial re-use. See rights and permissions. Published by BMJ.

To cite: Sejbaek T, Nielsen $\mathrm{HH}$, Penner $\mathrm{N}$, et al. J Neurol Neurosurg Psychiatry 2019:90:1324-1330.

\title{
Dimethyl fumarate decreases neurofilament light chain in CSF and blood of treatment naïve relapsing MS patients
}

\author{
Tobias Sejbaek (1) 1,2,3,4,5 Helle Hvilsted Nielsen, ${ }^{1,2,5}$ Natasha Penner, ${ }^{6}$ \\ Tatiana Plavina, ${ }^{6}$ Jason P Mendoza, ${ }^{6}$ Nellie Anne Martin, ${ }^{2}$ Maria Louise Elkjaer, ${ }^{1,2}$ \\ Mads Henrik Ravnborg, ${ }^{1}$ Zsolt Illes (i) 1,2,5
}

\section{ABSTRACT \\ Objectives In a prospective phase IV trial of the} first-line oral treatment dimethyl fumarate (DMF), we examined dynamics of neurofilament light (NFL) chain in serum, plasma and cerebrospinal fluid (CSF) samples collected over 12 months from relapsing-remitting multiple sclerosis (RRMS) patients. NFL changes were related to disease activity.

Methods We examined NFL levels by single-molecule array in 88 CSF, 348 plasma and 131 sera from treatment-naïve RRMS patients $(n=52)$, healthy controls $(n=23)$ and a placebo group matched by age, sex and NFL $(n=52)$. Plasma/sera were collected at baseline, and 1, 3, 6 and 12 months after DMF. CSF samples were collected at baseline and 12 months after DMF.

Results NFL concentration in CSF, plasma and serum correlated highly ( $p<0.0001$ for all), but plasma levels were only $76.9 \%$ of paired serum concentration. After 12 months of DMF treatment, NFL concentration decreased by $73 \%, 69 \%$ and $55 \%$ in the CSF, serum and plasma ( $p<0.0001$, respectively). Significant reduction in blood was observed after 6 and 12 months treatment compared with baseline $(p<0.01$ and $p<0.0001$, respectively) and to placebo $(p<0.0001)$. Patients with NFL above the $807.5 \mathrm{pg} / \mathrm{mL}$ cut-off in CSF had 5.0-times relative risk of disease activity $(p<0.001)$.

Conclusions This study provides Class II evidence that first-line DMF reduces NFL in both blood and CSF after 6 months and normalises CSF levels in $73 \%$ of patients. High NFL concentration in CSF after a year reflected disease activity. NFL levels were higher in serum than in plasma, which should be considered when NFL is used as a biomarker.

\section{INTRODUCTION}

Levels of the cytoskeleton protein neurofilament light (NFL) are increased in the cerebrospinal fluid (CSF) and blood in diseases associated with axonal damage. ${ }^{1}$ If measured by sensitive methods, blood and CSF concentrations of NFL correlate. ${ }^{2-4}$

Studies have shown that in the blood and CSF of patients with multiple sclerosis (MS), NFL concentration is elevated at the time of diagnosis compared with healthy controls (HC) and is associated with disease severity and prognosis. ${ }^{5}$ NFL levels are also increased during relapse and when new lesions are detected by MRI. ${ }^{67}$ Treatment with highly effective disease modifying therapies (DMTs), such as natalizumab, fingolimod, cladribine, daclizumab and alemtuzumab resulted in significant reduction in NFL in the CSF and blood. ${ }^{8-13}$ Switch from injectables to fingolimod reduced NFL concentration in the plasma, and switch to rituximab decreased NFL levels in the CSF. ${ }^{14}{ }^{15}$ In patients treated with natalizumab, such reduction in NFL in the CSF correlated with brain atrophy changes. ${ }^{16}$ Therefore, NFL has been suggested as a supplementary biomarker in the management of MS. ${ }^{17}$

Prospective studies that investigate changes of NFL in the blood and CSF during treatment with first-line DMTs are lacking. In this prospective open label phase IV study, we investigated dynamic changes of NFL concentration in parallel samples of serum, plasma and CSF taken from newly diagnosed treatment-naïve relapsing-remitting multiple sclerosis (RRMS) patients after treatment with dimethyl fumarate (DMF). We also compared NFL levels in MS patients before and after DMF treatment to healthy controls and placebo patients from the randomised phase III trial: Pegylated interferon $\beta-1 \mathrm{a}$ for relapsing-remitting multiple sclerosis (ADVANCE). ${ }^{18}$

\section{METHODS}

\section{Study design}

TREMEND (Tecfidera in Relapsing-Remitting Multiple Sclerosis: Endothelial Dysfunction) was a prospective open label phase IV trial which enrolled newly diagnosed MS patients with RRMS from March 2014 till August 2016 (EudraCT 2014 000254-11) (see online supplementary figure 1). TREMEND was a study designed to identify potential biomarkers in DMF-treated MS patients. The primary and secondary objectives were the change in the levels of biomarkers from baseline by month 12 and 24 in subjects with RRMS receiving DMF. Analysing NFL was one of the biomarker endpoints, and part of the original protocol.

We included untreated (naïve) newly diagnosed MS patients with RRMS according to the McDonald 2010 criteria (table 1). ${ }^{19}$ All patients had oligoclonal bands (OCBs) in the CSF $(n=52)$. The reason for including only patients with positive OCBs was to ensure that participants had active disease since absence of OCBs has been described a marker of low disease activity ${ }^{20}$ and support the 


\begin{tabular}{|c|c|c|c|c|}
\hline & $\begin{array}{l}\text { MS patients } \\
\text { TREMEND } \\
\text { DMF } \\
( \pm \text { SD) }\end{array}$ & $\begin{array}{l}\text { MS patients } \\
\text { ADVANCE } \\
\text { placebo } \\
( \pm \text { SD) }\end{array}$ & $\begin{array}{l}\text { Healthy controls } \\
( \pm \text { SD })\end{array}$ & $P$ value \\
\hline Age (years) & $34.1( \pm 8.7)$ & $33.5( \pm 6.5)$ & $38.2( \pm 11.2)$ & ns \\
\hline $\begin{array}{l}\text { Female/male } \\
(\%)\end{array}$ & $86.6 \%$ & $90 \%$ & $89.2 \%$ & ns \\
\hline $\begin{array}{l}\text { Baseline CSF } \\
\text { NFL (pg/mL) }\end{array}$ & $2368( \pm 1947)$ & $\mathrm{N} / \mathrm{A}$ & $416.8( \pm 191.2)$ & $P<0.0001$ \\
\hline $\begin{array}{l}\text { Baseline } \\
\text { plasma NFL }\end{array}$ & $16.4( \pm 14.4)$ & $17.5( \pm 14.0)$ & $7.3( \pm 3.0)$ & $P<0.0001$ \\
\hline \multicolumn{5}{|c|}{$\begin{array}{l}\text { TREMEND (Tecfidera in Relapsing-Remitting Multiple Sclerosis: Endothelial } \\
\text { Dysfunction) ( } \mathrm{n}=52 \text { ) and ADVANCE (Pegylated interferon } \beta-1 \text { a for relapsing- } \\
\text { remitting multiple sclerosis) }(\mathrm{n}=48)^{18} ; \text { mean } \pm \text { SD is shown. P values represent } \\
\text { difference between healthy controls }(\mathrm{n}=23) \text { versus patients in the ADVANCE and } \\
\text { TREMEND trial, respectively. There was no significant difference between the } \\
\text { ADVANCE and TREMEND trial. } \\
\text { CSF, cerebrospinal fluid; DMF, dimethyl fumarate; MS, multiple sclerosis; N/A, not } \\
\text { available; NFL, neurofilament light; } n s \text {, not significant. }\end{array}$} \\
\hline
\end{tabular}

inclusion of patients with MS even after a single clinical event. ${ }^{21}$ Patients were recruited from two MS clinics of the MS Alliance of Southern Denmark (Odense University Hospital and Hospital of South West Jutland), but the clinical examination and sample preparation were performed by the same physician (TS) following identical protocols. We collected plasma and sera at baseline, and 1, 3, 6 and 12 months after DMF treatment. Expanded Disability Status Scale (EDSS) ${ }^{22}$ scoring was performed at the same time points and when clinical relapse was suspected. CSF was collected at baseline $(n=30)$ and optionally 12 months after treatment $(n=35)$. Altogether, we examined 65 CSF samples, 108 serum samples and 230 plasma samples. Patients had brain MRI at baseline, 3 to 6 months after initiation of DMF (re-baseline) and after 12 months table 1. Patients with relapses and/or new or enlarging T2-weighted lesions on MRI were defined as patients with disease activity (national guidelines). ${ }^{23}$

We also collected CSF, plasma and serum from an age-matched and sex-matched healthy control cohort $(n=23)$ : patients without neurological disease and neurological signs, and with normal MRI and CSF results (table 1).

Since placebo group could not be added to the TREMEND trial for ethical reasons, we added an MS control group $(n=48)$ treated with placebo in the ADVANCE trial (phase III trial of pegylated interferon $\beta-1 \mathrm{a}) .{ }^{18}$ These patients were matched to the TREMEND patients based on age, sex and baseline NFL levels (table 1). EDSS in the TREMEND and ADVANCE trial was also similar $(1.77 \pm 0.8,1.95 \pm 1.0)$.

Samples were collected following international guidelines for biobanking ${ }^{24}$; CSF, plasma and sera were centrifuged 30 to $60 \mathrm{~min}$ after collection at $2000 \mathrm{~g}$ for $10 \mathrm{mins}$ at $20^{\circ} \mathrm{C}$ (room temperature), aliquoted in $500 \mu \mathrm{l}$ Sarstedt polypropylene tubes and stored at $-80^{\circ} \mathrm{C}$ until processing. NFL concentrations in sera, plasma and CSF were measured at Quanterix (Lexington, Massachusetts, USA) by using the commercially available sensitive assay single-molecule array as described by the manufacturer.

\section{Classification of evidence}

This interventional study provides Class II evidence that NFL is significantly reduced in both blood and CSF after 6 and 12 months treatment with DMF.
Standard protocol approvals, registrations, patient consents and monitoring

All patients and healthy controls gave written and oral consent. The study was monitored according to the national laws by the unit for good clinical practice at the hospital.

\section{Data protection}

This study was approved by the Danish Data Protection Agency (journal no. 17/12684).

\section{Data availability}

Anonymised data will be shared on request from any qualified investigator under approval from the Danish Data Protection Agency.

\section{Statistical analyses}

We described baseline characteristics with means and SDs for continuous variables and percentages for binary variables.

Linear fit regression was performed using Spearman linear fit regression to calculate coefficients and linearity between NFL in CSF, plasma and serum. Data was checked for normality using D'Agostino \& Pearson normality test.

Receiver operating characteristic (ROC) analysis was performed to identify cut-offs of NFL concentration in blood and CSF that differentiate healthy controls and MS patients, and define their specificity and sensitivity.

We used ordinary one-way analysis of variance and HolmSidak's multiple comparisons test in normal distributed data. In the absence of Gaussian distribution, non-parametric analysis was performed with Kruskal-Wallis test and Dunn's multiple comparisons.

To analyse relative risk between high (above cut-off) and low (below cut-off) NFL levels in CSF and blood, we used Fisher's exact test. Statistical analysis and data management were performed using GraphPad Prism 7.

\section{RESULTS}

NFL levels correlate in body fluids and are the lowest in plasma

To examine NFL in body fluids obtained from MS and HC, we first compared NFL levels in the CSF to paired plasma and serum samples $(\mathrm{n}=88)$; plasma levels were also compared with the paired serum $(n=129)$ (figure 1$)$. We observed the strongest correlation between plasma and serum levels $(\mathrm{p}<0.0001$; Spearman r: 0.96). CSF levels highly correlated with both plasma ( $<<0.0001$; Spearman r: 0.79) and serum NFL levels $(p<0.0001$; Spearman $r: 0.72)$. We also determined the ratio between CSF, serum and plasma NFL concentrations. NFL levels in plasma were on average $0.45 \%$ of NFL in the CSF, serum NFL levels were on average $0.52 \%$ of CSF levels and plasma NFL levels were on average $76.9 \%$ of NFL levels in the serum.

\section{NFL levels are higher in serum, plasma and CSF of newly-} diagnosed MS patients compared to HC

Next, we compared NFL concentration in serum, plasma and CSF obtained from treatment-naïve, newly diagnosed MS patients to HC. NFL levels in the CSF were higher in MS patients $(2368 \pm 1947 \mathrm{pg} / \mathrm{mL})$ compared with $\mathrm{HC}(417 \pm 191 \mathrm{pg} / \mathrm{mL})$ $(\mathrm{p}<0.001)$ (figure 2A). Plasma NFL levels were also higher in plasma $(16.4 \pm 14.4 \mathrm{pg} / \mathrm{mL})$ and in sera $(25.0 \pm 43.9 \mathrm{pg} / \mathrm{mL})$ of the untreated MS patients (baseline) compared with healthy controls $(7.3 \pm 3.0)(\mathrm{p}<0.01$ and $\mathrm{p}<0.0001$, respectively) (figure $2 \mathrm{~B})$. 

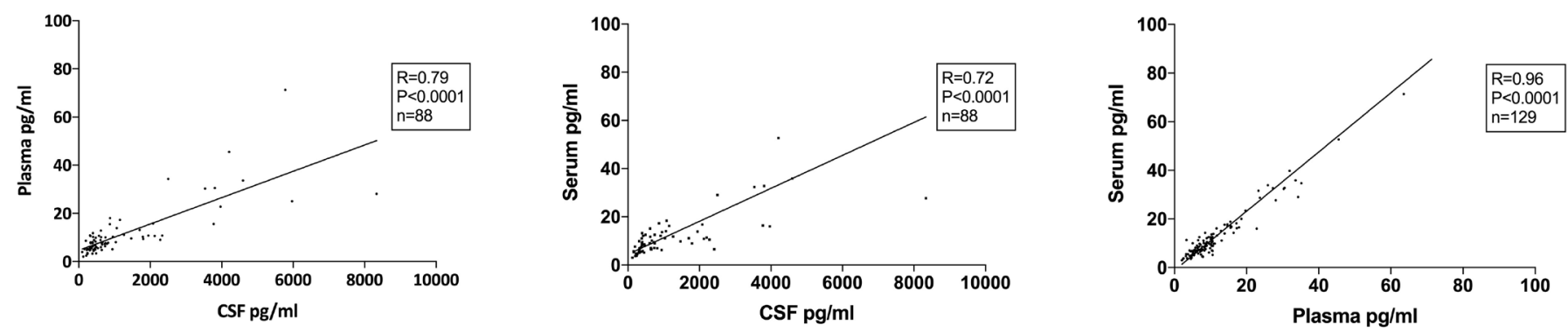

Figure 1 Correlation between NFL levels in CSF, plasma and serum. Linear regression analysis of respective body fluids, depicting Spearman $r$ value and levels of significance. CSF, cerebrospinalfluid; NFL, neurofilament light. $n_{\text {CSF }}=88, n_{\text {plasma }}=129, n_{\text {serum }}=129$.

\section{Cut-off concentrations discriminate between HC and MS patients}

In order to identify cut-offs that discriminate NFL levels in HC from MS patients, we performed ROC analysis (figure 3). ROC analysis showed an area under curve 0.933 (95\% CI 0.865 to 1.002, $\mathrm{p}<0.0001)$ in the CSF $(\mathrm{n}=53), 0.757$ (95\% CI 0.645 to $0.868, \mathrm{p}<0.001)$ in the plasma $(\mathrm{n}=70)$ and $0.778(95 \% \mathrm{CI}$ 0.669 to $0.888, \mathrm{p}<0.001)$ in the serum $(\mathrm{n}=68)$. To discriminate between MS patients and healthy controls, a cut-off concentration of $807.5 \mathrm{pg} / \mathrm{mL}$ in the CSF showed $100 \%$ specificity and $80 \%$ sensitivity; a cut-off concentration of $13.0 \mathrm{pg} / \mathrm{mL}$ in the plasma showed $100 \%$ specificity and $47 \%$ sensitivity and a cutoff concentration of $15.6 \mathrm{pg} / \mathrm{mL}$ in the serum showed $100 \%$ specificity and $43.2 \%$ sensitivity. Using other cut-off values did not improve overall sensitivity and specificity (data not shown).

By comparing individual CSF samples with increased NFL (ie, above cut-off) to paired plasma and serum, we found that NFL was below cut-off, that is, in the normal range in $43.3 \%$ of sera and $35.5 \%$ of plasma samples.
Continuous decline of NFL levels after treatment with DMF We next examined the change of NFL levels after DMF treatment in serum, plasma and CSF samples. After 1 year treatment, NFL levels were reduced by $1340 \mathrm{pg} / \mathrm{mL}$ in the CSF $(\mathrm{p}<0.0001)$, by $17.3 \mathrm{pg} / \mathrm{mL}$ in the serum $(\mathrm{p}<0.0001)$ and by $9.3 \mathrm{pg} / \mathrm{mL}$ in the plasma $(\mathrm{p}<0.01)$. These changes corresponded to a reduction of $73 \%, 69 \%$ and $57 \%$ in NFL levels in the CSF, serum and plasma, respectively. All sera samples, $96 \%$ of plasma samples, and $72 \%$ of CSF samples showed levels similar to healthy controls, that is below cut-off after 1 year treatment (figures 2C,D and 4).

In the placebo-treated group, plasma NFL levels remained unchanged, when samples collected at baseline $(17.5 \pm 14.0 \mathrm{pg} /$ $\mathrm{mL}$ ) were compared with samples obtained after 1 year placebo treatment $(16.6 \pm 14.0 \mathrm{pg} / \mathrm{mL})(\mathrm{p}>0.99)$ (figure $4 \mathrm{~A})$.

We also examined the dynamics and temporal profile of NFL reduction in the blood. After DMF treatment, plasma levels remained unchanged from baseline $(16.4 \pm 14.4 \mathrm{pg} / \mathrm{mL})$ to 1 month $(15.8 \pm 11.0 \mathrm{pg} / \mathrm{mL}, \mathrm{p}>0.99)$ and 3 months after treatment $(12.0 \pm 7.8 \mathrm{pg} / \mathrm{mL}, \mathrm{p}=0.78)$. Significant reduction in NFL
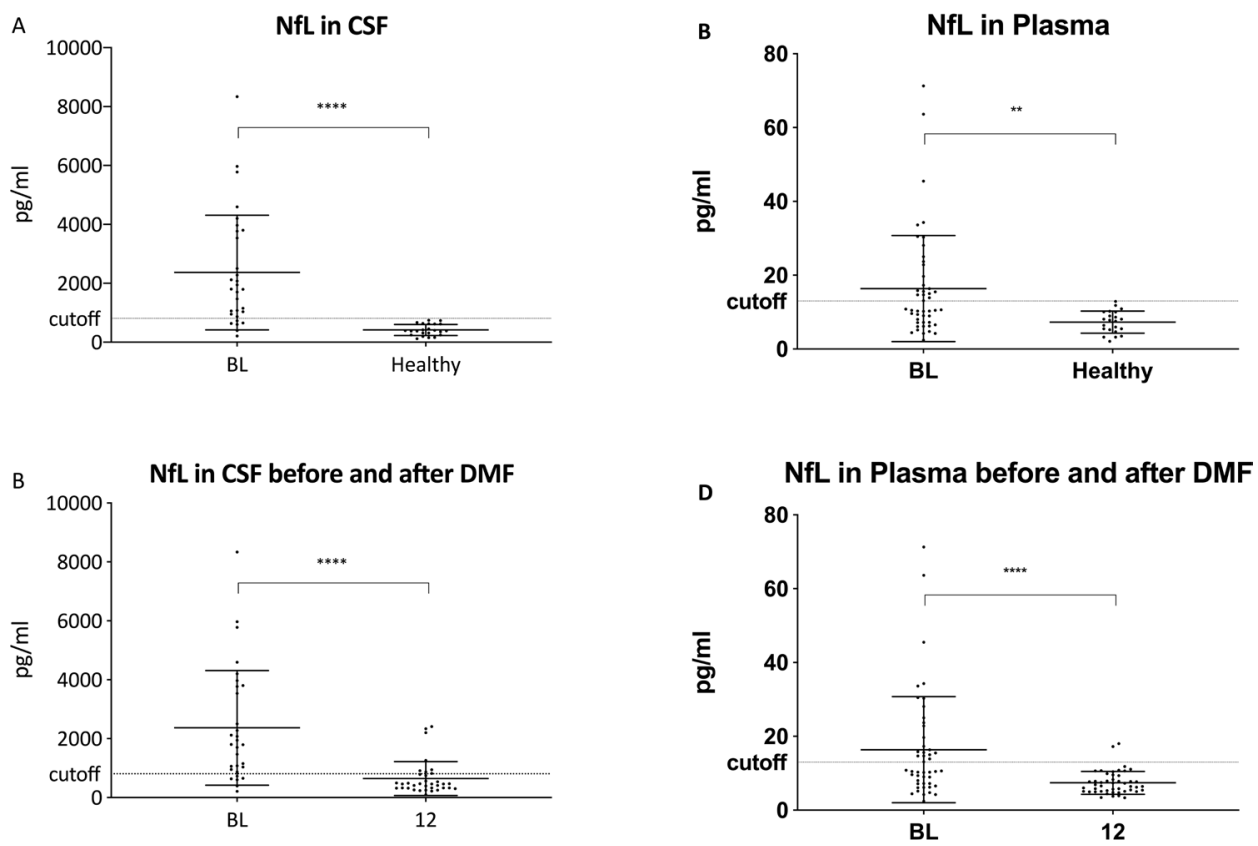

Figure $2 \mathrm{NFL}$ in the CSF and plasma of MS patients at baseline, after DMF treatment and in controls. NFL concentrations in MS patients at baseline compared with healthy controls (A, B) and after 12 months treatment with DMF (C, D). Cut-offs determined by receiver operating characteristic analysis (see figure 3) are shown with dotted line. Significance in difference between groups is shown: ${ }^{* *}: p<0.01,{ }^{* * *}$ : $p<0.0001$. BL, baseline; CSF, cerebrospinal fluid; DMF, dimethylfumarate; HC, healthy control; MS, multiple sclerosis; NFL, neurofilament light. $\mathrm{n}_{\mathrm{CSF} B \mathrm{BL}}=30, \mathrm{n}_{\text {plasma BL }}=47, \mathrm{n}_{\mathrm{CSF} \text { Healthy }}=23, \mathrm{n}_{\text {plasma Healthy }}=22, \mathrm{n}_{\mathrm{CSF}}$ ${ }_{12}=35, n_{\text {plasma } 12}=47$. 

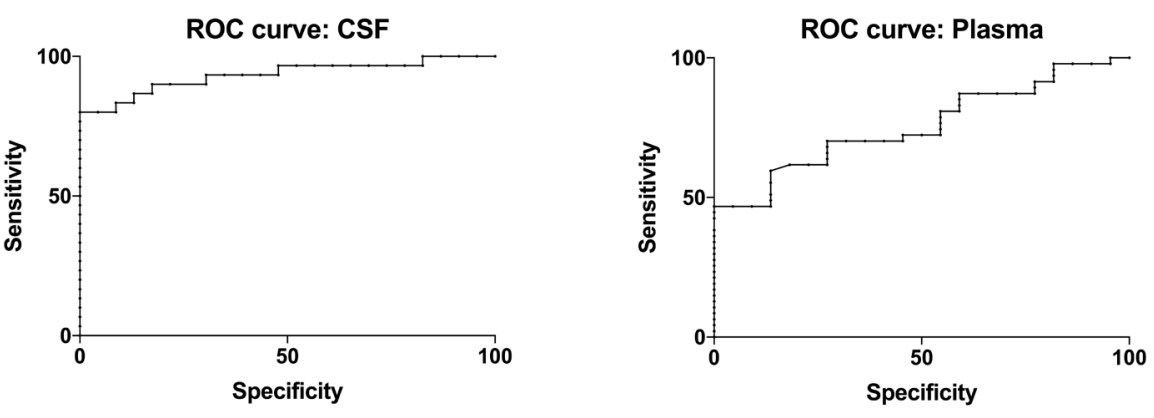

ROC curve: Serum

Figure 3 Sensitivity and specificity of NFL in the CSF, plasma and serum to discriminate multiple sclerosis patients from healthy controls. AUC for CSF NFL was $0.933(95 \% \mathrm{Cl} 0.865$ to $1.002, \mathrm{p}<0.0001)$, AUC for plasma NFL was 0.757 (95\% Cl 0.645 to $0.868, \mathrm{p}<0.001)$ and AUC for serum NFL 0.778 (95\% Cl 0.669 to $0.888, \mathrm{p}<0.001)$. CSF cut-off concentration $807.5 \mathrm{pg} / \mathrm{mL}$ showed $100 \%$ specificity and $80 \%$ sensitivity, plasma cut-off concentration $13.0 \mathrm{pg} / \mathrm{mL}$ showed $100 \%$ specificity and $47 \%$ sensitivity and serum cut-off concentration $15.6 \mathrm{pg} / \mathrm{mL}$ showed $100 \%$ specificity and $43.2 \%$ sensitivity. AUC, area under curve; CSF, cerebrospinal fluid; NFL, neurofilament light; $R O C$, receiver operating characteristic. $n_{\text {CSF }}=53, n_{\text {serum }}=68, n_{\text {plasma }}=70$.

plasma concentration was observed after $6(9.1 \pm 5.7 \mathrm{pg} / \mathrm{mL}$ $\mathrm{p}<0.01)$ and 12 months $(7.4 \pm 3.1 \mathrm{pg} / \mathrm{mL}, \mathrm{p}<0.0001)$ treatment with DMF. NFL concentration in the plasma of MS patients became similar to that of $\mathrm{HC}$ after 6 and 12 months treatment (figure 4A). NFL concentration in the serum showed similar dynamics: concentration reached the cut-off after 3 months treatment similar to plasma, became similar to HS and showed a significant reduction from baseline $(\mathrm{p}<0.0001)$ after 1 year of DMF (figure 4B). After 1 year, NFL levels showed a pattern of decline and below cut-off in 16 out of 22 CSF samples $(73 \%)$ (figure 5A).

High NFL levels after one year treatment with DMF are associated with disease activity

We also investigated, if disease activity during the first and second year after DMF was associated with change in NFL levels during the first year of treatment. Clinical data before and after DMF are presented in online supplementary table 1.

First we examined, if disease activity, that is, relapses and/ or new or enlarging T2-weighted lesions on MRI during DMF treatment was associated with increased NFL levels after 1 year of treatment. NFL levels were below cut-off in the CSF in $72.8 \%$ of $22 \mathrm{MS}$ patients (figure $5 \mathrm{~A}$ ), and below the cut-off in the plasma in $96.2 \%$ of $52 \mathrm{MS}$ patients after 1 year treatment, respectively. Patients with NFL in the CSF above cut-off $(807.5 \mathrm{pg} / \mathrm{mL})$ after 1 year of treatment had a relative risk of 5.0 of relapse and/or new T2-weighted lesions on MRI $(\mathrm{p}<0.001)$ during the first year of treatment (figure $5 \mathrm{~B}$ ). Patients with disease activity and NFL levels above cut-off in CSF at 12 months had disease activity between months 4 to 12 and patients with disease activity between month 0 to 4 were below cut-off in CSF after 12 months treatment with DMF (figure 5C). Disease activity during the second year of DMF was not in association with NFL levels below or above with cut-off in CSF $(p=0.66)$. Associations of disease activity with NFL levels in the blood could not be found in the first and second year of DMF treatment. There was no significant difference between the subgroups with and without disease activity at any time point (see online supplementary figure 2).

\section{DISCUSSION}

We determined NFL levels in the CSF, serum and plasma during and at the end of 1 year treatment with DMF in a prospective phase IV trial that enrolled newly diagnosed, treatmentnaive relapsing MS patients (see online supplementary figure 1). Results were compared with a healthy control group and a
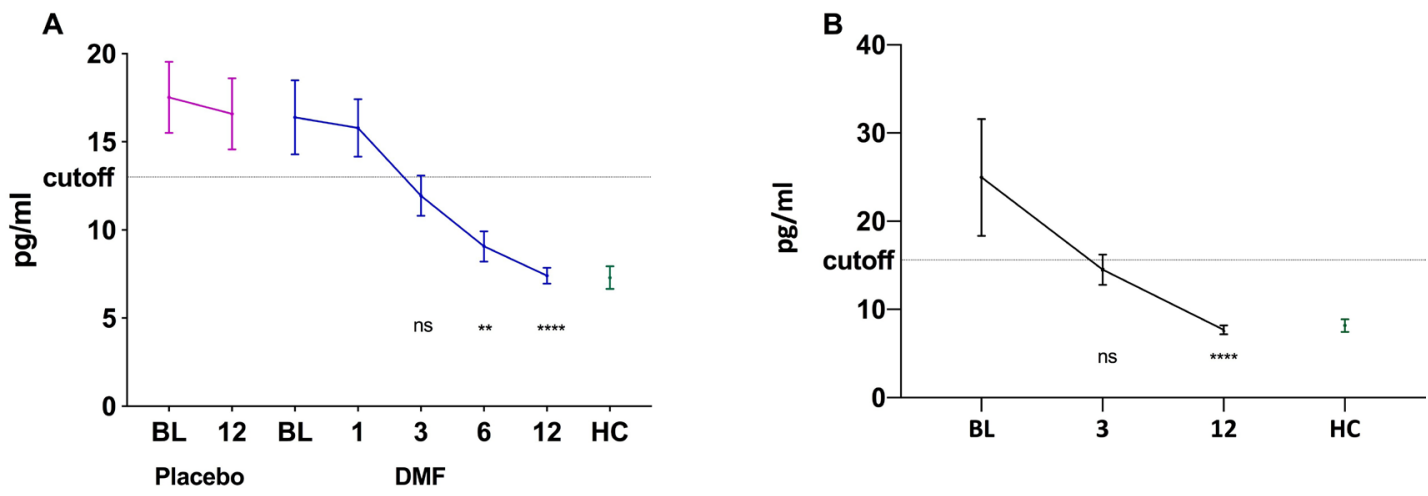

Figure 4 Dynamics of plasma and serum NFL concentrations in MS patients treated with placebo and/or DMF compared with healthy controls. A: Repeated plasma NFL levels in MS patients treated with DMF in the TREMEND trial (blue) and in age-matched and sex-matched placebo treated group in ADVANCE trial (purple) at baseline and at 1 year follow-up. Numbers at $x$ axis indicate follow-up in month. Concentration in healthy controls (green) from the TREMEND trial is also indicated. $n_{\text {placebo BL }}=48, n_{D M F}=47, n_{H C}=22 B$ : Repeated serum NFL levels in MS patients treated with DMF in the TREMEND trial. Numbers at $x$ axis indicate follow-up in month. Concentration in healthy controls (green) from the TREMEND trial is also indicated. $n_{\text {placebo }}=48$, $n_{D M F}=47, n_{H C}=22$ Mean values and SEM are shown at each time point. Significance in difference between baseline and follow-up is shown: ${ }^{* *} p<0.01$, $* * * * 00.0001$. ADVANCE, Pegylated interferon $\beta$-1afor relapsing-remitting multiple sclerosis; BL, baseline; DMF, dimethyl fumarate; HC, healthy control;MS, multiple sclerosis; NFL, neurofilament light; ns, not significant; TREMEND, Tecfidera in Relapsing-Remitting Multiple Sclerosis:Endothelial Dysfunction. 

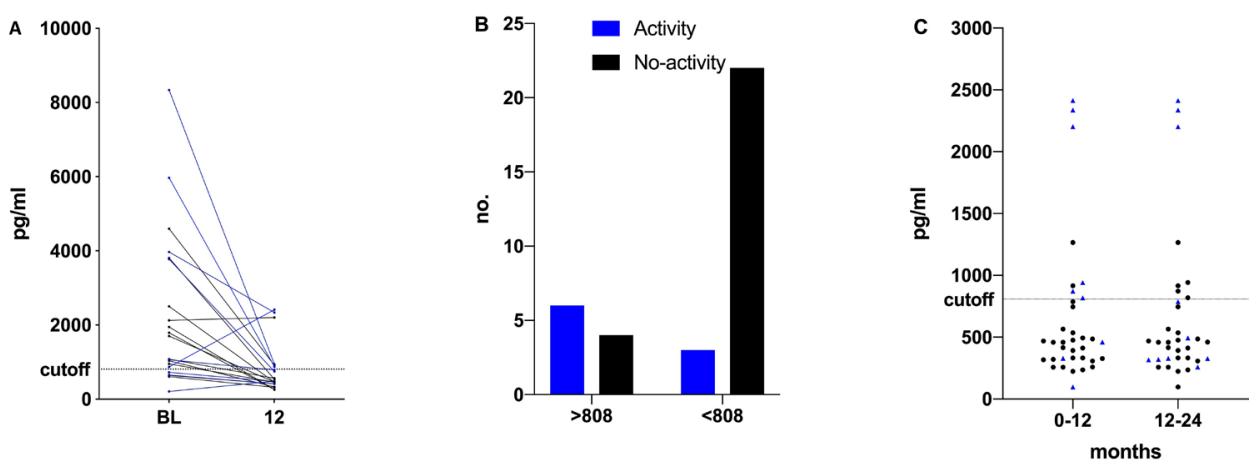

Figure 5 CSF NFL concentrations in MS patients before and after treatment with DMF and related to disease activity. Activity within the first year was defined by relapses and/or new/enlarging white matter lesions on MRI. Blue colour indicates disease activity and black colour stable disease. (A) Paired CSF samples from MS patient before and after DMF treatment $(n=22)$. Cut-off determined by ROC analysis are shown with dotted line. Blue lines are patients having disease activity within the first year. (B) Depicts patient with disease activity (blue) and stable disease (black) within the first year of DMF in groups stratified by NFL values below and above cut-offs after 1 year DMF treatment $(n=35)$. (C) Disease activity within 0 to 12 months and 12 to 24 months and NFL values in CSF after 12 months of DMF treatment $(n=35)$. Patients with disease activity and NFL levels above cut-off in CSF at 12 months had disease activity between months 4 to 12 and patients with disease activity between month 0 to 4 were below cut-off in CSF after 12 months treatment with DMF. Blue triangles indicate disease activity and black indicate stable disease. BL, baseline; CSF, cerebrospinal fluid; DMF, dimethyl fumarate; MS, multiple sclerosis; NFL, neurofilament light; ROC, receiver operating characteristic.

placebo control group from a phase III trial on pegylated interferon $\beta-1$ a (ADVANCE). Although a randomised placebo group in our TREMEND trial would have been more optimal, such phase IV trials cannot be approved in Denmark for first-line DMTs. The placebo-treated group from the ADVANCE trial was matched to the DMF-treated patients in TREMEND based on age, sex and NFL levels, and EDSS at baseline was also similar between the two trials. We also examined the dynamics of NFL in the serum and plasma during 1 year of DMF treatment in MS patients, and compared the sensitivity of blood and CSF NFL for detection of disease activity by using paired samples.

This study showed that NFL values in the CSF were approximately 200 times higher than in the plasma and serum, and thus support results seen in other MS cohorts. ${ }^{471525}$ We found the highest correlation between plasma and serum, with plasma demonstrating 23\% lower NFL values than serum (figure 1). Samples were collected at the same time following international guidelines; therefore, a systematic bias is unlikely. Difference between serum and plasma are known for other biomarkers, since clotting factor and tube material may affect the composition of the two body fluids. ${ }^{26}$ The differences between NFL levels in plasma and serum were significant and should be considered when biomarker outcomes in peripheral blood are planned in future studies, for example, when designing trials with either plasma or serum NFL as outcomes.

We also identified NFL cutoffs with ROC analysis to discriminate between patients with newly diagnosed treatment naïve RRMS and healthy controls (figures 2 and 3). The identified blood cut-offs were similar to previous data, ${ }^{27}$ but some other studies demonstrated a wide range of mean NFL from 9 to $36 \mathrm{pg} /$ $\mathrm{mL}$ in blood. ${ }^{7252829}$ Those studies however did not define cutoff based on ROC analysis, and the enrolled patient populations were heterogeneous regarding treatment and clinical disease course. We found a CSF cut-off of $807.5 \mathrm{pg} / \mathrm{mL}$, which is similar to results published within the last 5 years; these studies detected cut-offs above and below $1000 \mathrm{pg} / \mathrm{mL}$ with mean values from 463 to $2500 \mathrm{pg} / \mathrm{mL}^{11127283031}$ (figure 3).

Observing differences between newly diagnosed untreated MS patients and HC, we next examined the impact of 1 year DMF treatment on NFL concentration in the serum/plasma/CSF compared with a placebo group. In addition, we investigated the dynamics of NFL changes and the temporal profile of normalisation in the plasma after DMF. We found a continuous decrease of NFL in the blood after starting DMF that became significant after 6 months treatment and continued declining up to 12 months (figure 4). Such decrease in the blood was also supported by a significant similar reduction of NFL in the CSF after 12 months (figure 2). The trend of reduction was observed after 3 months (figure 4), the time point when clinical effects became significant in both the DEFINE (The Determination of the Efficacy and Safety of Oral Fumarate in Relapsing-Remitting MS study) and CONFIRM (The members of the Comparator and and Oral Fumarate in Relapsing Remitting Multiple Sclerosis) trials. $^{32} 33$

The observed dynamics of decline are related to the effect of treatment and not to the natural course, as we did not see any decline in patients treated with placebo for 1 year. The relative larger reduction in serum NFL compared with plasma NFL could suggest that serum is more useful in detecting changes, but this should be addressed by specifically designed studies.

Our data indicates that DMF used as a first-line treatment has similar potential in reducing NFL levels in the blood and CSF compared with highly effective DMTs even though this cohort demonstrated mild disease burden prior to initiation of treatment. A few studies have already shown that natalizumab, ${ }^{89222434}$ fingolimod, ${ }^{1011} 1528$ cladribine $^{35}$ daclizumab $^{36}$ and alemtuzumab ${ }^{13}$ are able to reduce NFL levels in both the CSF and/or blood. ${ }^{8} 101113153435$

Our data would suggest that NFL may be used as biomarker to monitor treatment responses during the first treatment of MS. Therefore, we also examined, if higher NFL levels after 1 year of DMF treatment was associated with higher disease activity. 5891528 We were able to directly compare blood and CSF NFL levels in detecting clinical activity by utilising paired serum/plasma-CSF samples. By using the calculated NFL cut-off in CSF $(807.5 \mathrm{pg} / \mathrm{mL})$, we could retrospectively identify patients who had experienced disease activity during the first year of treatment (figure 5). However, NFL levels in paired plasma and serum were unable to identify the same patients. In addition NFL levels in CSF at 12 months did not predict disease activity in month 12 to 24. Further trials designed for examination of dynamics and clinical significant difference of NFL are needed. 
This discrepancy between blood and CSF measurement probably reflects the lower NFL concentration in the systemic compartment compared with the CSF, possibly because CSF is closer to the damaged tissue. We found that despite the increased NFL concentration in the CSF, $13.6 \%$ and $22.1 \%$ of the respective paired serum/plasma samples showed normal values. Another study also showed higher sensitivity of CSF NFL for detecting disease activity compared with serum. ${ }^{27}$ This lower sensitivity of NFL measurement in the peripheral compartment may limit the value of evaluating clinical activity, although blood is a more accessible body fluid than CSF and can be repeatedly analysed. Since serial lumbar puncture is not possible in an ordinary clinical setting, modifications in the assay that increases sensitivity in the plasma are needed. Alternatively, regular monitoring with blood samples at predefined intervals, for example 2 to 3 months should be necessary in order to avoid misinterpreting normal plasma values at time points, when CSF still shows mild elevation suggesting active disease. Further studies are necessary to understand the required frequency of such laboratory tests. ${ }^{7}$

Our study is not without limitations. MRI lesions with gadolinium enhancement have not been included in the study, and we considered disease activity based on clinical relapses and new/enlarging T2 lesions. The placebo group was not part of the TREMEND trial for ethical reasons, and was added based on matching age, sex and baseline NFL levels. We did not find association between NFL levels and EDSS or age in univariate analysis (data not shown), but this is related to the trial design, and the cohort with narrow age and EDSS range in newly diagnosed, treatment-naïve patients.

In conclusion, we demonstrate that DMF as first-line treatment reduces NFL levels in both the blood and CSF, and such reduction becomes significant after 6 months. NFL levels in CSF above the calculated cut-off reflected disease activity within the first year but did not predict disease activity in the second year. NFL levels in plasma are lower than in paired serum samples, and this should be considered during future trial design. Additional studies are needed to implement NFL as a clinical monitoring tool in patients with MS receiving DMT and determine the minimum frequency of blood NFL to detect subclinical disease activity.

Contributors TS contributed with: concept and design, acquisition, analysis and interpretation of data, drafting of the manuscript, revision of the manuscript, critical revision of the manuscript for important intellectual content, statistical analysis. HHN contributed with: analysis, acquisition, interpretation of data and revision of manuscript. NP contributed with: analysis, interpretation of data and revision of manuscript. TP contributed with: analysis, interpretation of data and revision of manuscript. JPM contributed with: analysis, interpretation of data and revision of manuscript. NAM contributed with: acquisition of data and manuscript. MLE contributed with: acquisition of data and manuscript. MHR contributed with concept and design, acquisition, analysis, interpretation, manuscript and revision of manuscript. ZI contributed with concept and design, writing the protocol of the trial, obtaining the necessary research grants, analysis and interpretation of data, drafting of the manuscript, revision of the manuscript, critical revision of the manuscript for important intellectual content, study supervisor.

Funding This trial was funded by research grants from Biogen (TREMEND to ZI and TS), University of Southern Denmark (14/24200 to TS and ZI), Odense University Hospital (5798002573633 to TS and ZI) and The Danish Multiple Sclerosis Society (R367-A25341 to ZI).

Competing interests TS has served on scientific advisory boards, received support for congress participation, received speaker honoraria and received research support from Biogen and Novartis. NP is employee of and holds stock/ stock options in Biogen. TP is employee of and holds stock/stock options in Biogen. JPM is employee of and holds stock/stock options in Biogen. HHN has served on scientific advisory boards, received support for congress participation, received speaker honoraria and received research support from Biogen, Merck-Serono, Lundbeck and Novartis. Zl has served on scientific advisory boards, served as a consultant, received support for congress participation, received speaker honoraria and received research support from Biogen, Merck-Serono, Sanofi-Genzyme, Lundbeck and Novartis.

Patient consent for publication Obtained.

Ethics approval This study was approved by the regional Committee of Health Research Ethics (S-20140015HLP/csf), the Danish Health and Medicines Authority (2014013769) and the European Clinical Trials Database (2014-000254-11).

Provenance and peer review Not commissioned; externally peer reviewed. Data availability statement Data are available upon reasonable request.

Open access This is an open access article distributed in accordance with the Creative Commons Attribution Non Commercial (CC BY-NC 4.0) license, which permits others to distribute, remix, adapt, build upon this work non-commercially, and license their derivative works on different terms, provided the original work is properly cited, appropriate credit is given, any changes made indicated, and the use is non-commercial. See: http://creativecommons.org/licenses/by-nc/4.0/.

\section{ORCID iDs}

Tobias Sejbaek http://orcid.org/0000-0002-7682-2188

Zsolt llles http://orcid.org/0000-0001-9655-0450

\section{REFERENCES}

1 Norgren N, Rosengren L, Stigbrand T. Elevated neurofilament levels in neurological diseases. Brain Res 2003:987:25-31.

2 Deisenhammer F, Egg R, Giovannoni G, et al. EFNS guidelines on disease-specific CSF investigations. Eur J Neurol 2009;16:760-70.

3 Khalil M, Teunissen CE, Otto M, et al. Neurofilaments as biomarkers in neurological disorders. Nat Rev Neurol 2018;14:577-89.

4 Kuhle J, Barro C, Andreasson U, et al. Comparison of three analytical platforms for quantification of the neurofilament light chain in blood samples: ELISA electrochemiluminescence immunoassay and Simoa. Clin Chem Lab Med 2016;54:1655-61.

5 Lycke JN, Karlsson JE, Andersen O, et al. Neurofilament protein in cerebrospinal fluid: a potential marker of activity in multiple sclerosis. J Neurol Neurosurg Psychiatry 1998:64:402-4.

6 Barro C, Benkert P, Disanto G, et al. Serum neurofilament as a predictor of disease worsening and brain and spinal cord atrophy in multiple sclerosis. Brain 2018;141:2382-91

7 Disanto G, Barro C, Benkert P, et al. Serum neurofilament light: a biomarker of neuronal damage in multiple sclerosis. Ann Neurol 2017;81:857-70.

8 Axelsson M, Malmeström C, Gunnarsson M, et al. Immunosuppressive therapy reduces axonal damage in progressive multiple sclerosis. Mult Scler 2014;20:43-50.

9 Gunnarsson M, Malmeström C, Axelsson M, et al. Axonal damage in relapsing multiple sclerosis is markedly reduced by natalizumab. Ann Neurol 2011;69:83-9.

10 Kuhle J, Disanto G, Lorscheider J, et al. Fingolimod and CSF neurofilament light chain levels in relapsing-remitting multiple sclerosis. Neurology 2015;84:1639-43.

11 Novakova L, Axelsson M, Khademi M, et al. Cerebrospinal fluid biomarkers of inflammation and degeneration as measures of fingolimod efficacy in multiple sclerosis. Mult Scler 2017:23:62-71.

12 Kuhle J, Kropshofer $\mathrm{H}$, Haering DA, et al. Blood neurofilament light chain as a biomarker of MS disease activity and treatment response. Neurology 2019;92:e1007-15.

13 Hyun JW, Kim Y, Kim G, et al. Longitudinal analysis of serum neurofilament light chain: a potential therapeutic monitoring biomarker for multiple sclerosis. Mult Scler 2019:1352458519840757.

14 de Flon P, Gunnarsson M, Laurell K, et al. Reduced inflammation in relapsing-remitting multiple sclerosis after therapy switch to rituximab. Neurology 2016:87:141-7.

15 Piehl F, Kockum I, Khademi M, et al. Plasma neurofilament light chain levels in patients with MS switching from injectable therapies to fingolimod. Mult Scler 2018;24:1046-54

16 Mellergård J, Tisell A, Blystad I, et al. Cerebrospinal fluid levels of neurofilament and tau correlate with brain atrophy in natalizumab-treated multiple sclerosis. Eur J Neurol 2017;24:112-21.

17 Cai L, Huang J. Neurofilament light chain as a biological marker for multiple sclerosis: a meta-analysis study. Neuropsychiatr Dis Treat 2018;14:2241-54.

18 Calabresi PA, Kieseier BC, Arnold DL, et al. Pegylated interferon beta-1a for relapsingremitting multiple sclerosis (ADVANCE): a randomised, phase 3, double-blind study. Lancet Neurol 2014:13:657-65.

19 Polman CH, Reingold SC, Banwell B, et al. Diagnostic criteria for multiple sclerosis: 2010 revisions to the McDonald criteria. Ann Neurol 2011;69:292-302.

20 Tintore M, Rovira Àlex, Río J, et al. Defining high, medium and low impact prognostic factors for developing multiple sclerosis. Brain 2015;138:1863-74.

21 Thompson AJ, Banwell BL, Barkhof F, et al. Diagnosis of multiple sclerosis: 2017 revisions of the McDonald criteria. Lancet Neurol 2018;17:162-73.

22 Kurtzke JF. Rating neurologic impairment in multiple sclerosis: an expanded disability status scale (EDSS). Neurology 1983;33:1444-52. 
23 RADS. The Danish Council for the use of expensive Hospital medicines, 2016 Available: http://www.regioner.dk/media/2830/radsfolder-engelsk.pdf

24 Teunissen CE, Petzold A, Bennett JL, et al. A consensus protocol for the standardization of cerebrospinal fluid collection and biobanking. Neurology 2009;73:1914-22

25 Disanto G, Adiutori R, Dobson R, et al. Serum neurofilament light chain levels are increased in patients with a clinically isolated syndrome. J Neurol Neurosurg Psychiatry 2016:87:126-9.

26 Boeynaems J-M, De Leener A, Dessars B, et al. Evaluation of a new generation of plastic evacuated blood-collection tubes in clinical chemistry, therapeutic drug monitoring, hormone and trace metal analysis. Clin Chem Lab Med 2004;42:67-71.

27 Håkansson I, Tisell A, Cassel P, et al. Neurofilament levels, disease activity and brain volume during follow-up in multiple sclerosis. J Neuroinflammation 2018;15:209.

28 Novakova L, Zetterberg $\mathrm{H}$, Sundström P, et al. Monitoring disease activity in multiple sclerosis using serum neurofilament light protein. Neurology 2017;89:2230-7.

29 Kuhle J, Barro C, Disanto G, et al. Serum neurofilament light chain in early relapsing remitting MS is increased and correlates with CSF levels and with MRI measures of disease severity. Mult Scler 2016;22:1550-9.
30 Novakova L, Axelsson M, Malmeström C, et al. Reduced cerebrospinal fluid concentrations of oxysterols in response to natalizumab treatment of relapsing remitting multiple sclerosis. J Neuro/ Sci 2015;358:201-6.

31 Rosengren LE, Karlsson JE, Karlsson JO, et al. Patients with amyotrophic latera sclerosis and other neurodegenerative diseases have increased levels of neurofilament protein in CSF. J Neurochem 1996:67:2013-8.

32 Fox RJ, Miller DH, Phillips JT, et al. Placebo-controlled phase 3 study of oral BG-12 or glatiramer in multiple sclerosis. N Engl J Med 2012;367:1087-97.

33 Gold R, Kappos L, Arnold DL, et al. Placebo-controlled phase 3 study of oral BG-12 for relapsing multiple sclerosis. N Engl J Med 2012;367:1098-107.

34 Amor S, van der Star BJ, Bosca I, et al. Neurofilament light antibodies in serum reflect response to natalizumab treatment in multiple sclerosis. Mult Scler 2014:20:1355-62.

35 Yildiz 0, Mao Z, Adams A, et al. Disease activity in progressive multiple sclerosis can be effectively reduced by cladribine. Mult Scler Relat Disord 2018;24:20-7.

36 Komori M, Kosa P, Stein J, et al. Pharmacodynamic effects of daclizumab in the intrathecal compartment. Ann Clin Trans/ Neurol 2017:4:478-90 